\title{
Glycogen storage diseases of all types
}

\author{
Georg F. Hoffmann ${ }^{1}$ • P. A. Smit ${ }^{2}$ - B. Schoser ${ }^{3}$
}

Published online: 5 May 2015

(C) SSIEM 2015

Defects of glycogen metabolism are among the earliest recognized inborn errors of metabolism, first described in 1928 and 1929. When Schönheimer demonstrated in 1929 that an enzyme that degrades glycogen was missing in the liver of a patient suffering from what is now known as glycogenosis type 1 or von Gierke disease, Sir Archibald Garrod's concept that inborn errors of metabolism result from genetically determined enzyme deficiencies was proven for the first time. Since then, a multitude of disturbances of glycogen metabolism was defined, and virtually defects of all proteins involved in synthesis, transport, degradation of glycogen and its regulation have been discovered. The delineation of glycogenin-1 deficiency in 2010 marked the 20th monogenic defect in this field and was designated as glycogen storage disease type XV.

In the last two decades, progress in the understanding of inborn errors of metabolism has spearheaded the development of molecular medicine. Scientific and

Georg F. Hoffmann

georg.hoffmann@med.uni-heidelberg.de

P. A. Smit

GPASMIT@hetnet.nl

B. Schoser

bschoser@med.uni-muenchen.de

1 Department of Pediatrics, University of Heidelberg, Im Neuenheimer Feld 430, 69120 Heidelberg, Germany

2 Faculty of Medical Science, Paediatrics, Hanzeplein 1, 9713 GZ Groningen, The Netherlands

3 Friedrich-Baur-Institut, Neurologische Klinik, Klinikum der Universität München, Ziemssenstr. 1a, 80336 Munich, Germany technological advances translated into diagnostic and therapeutic medical progress can offer enormous benefit to patients suffering from inborn errors of metabolism, by preventing or reducing life-long burden and suffering. These disorders remain orphan entities, and the World Health Organisation and the European Union have announced genetic and orphan diseases as a major health challenge of the future. Progress in the field can still seem painfully slow. Metabolic medicine is evolving from a very personal individual interaction between one family and one doctor to a sophisticated team approach involving different medical specialties and associated professionals including biochemists, dieticians, nurses, psychologists, physiotherapists, social workers, speech therapists, teachers and, most importantly, patient organizations in worldwide networks.

In Heidelberg, medical experts and scientists met together with patients and their families for three intensive days for the International Glycogen Storage Disease Conference in November 2013. The Selbsthilfe Gruppe Deutschland Glykogenose e. V. (German GSD Patient Support Group) was the main local partner and host. Altogether, 29 patient associations were involved, alphabetically from the Acid Maltase Deficiency Association (AMDA) to the "Vereniging Volwassen Kinderen en Stofwisselingsziekten (VKS)". For the first time patients, families, clinicians and researchers were brought together to review and examine in depth the state of our current knowledge to transfer and discuss new knowledge and to develop and discuss ideas for the next steps in basic, translational and clinical research. Gaps were identified and collaborative research were initiated to address unmet medical needs of those affected. These 
three intense days went by very quickly. We are delighted that the essence of the conference could be distilled into 12 excellent manuscripts, again covering many aspects and types of glycogen storage diseases. They form the body of this special issue. We thank the authors, the reviewers and the staff of the Journal of Inherited Metabolic Disease for all their efforts and trust that this special issue will play its part in providing important information and as a stimulus to the different initiatives in countries all over the world to further advance and professionalize research and care for "Glycogen Storage Diseases of all Types", which was the motto of the conference.

Conflict of interest None. 